IFT-7/2000

hep-th/0003233

\title{
Anomaly cancellation in M-theory on orbifolds
}

\author{
Krzysztof A. Meissner and Marek Olechowski \\ Institute of Theoretical Physics \\ Warsaw University \\ Hoża 69, 00-681 Warszawa, Poland
}

\begin{abstract}
We present calculation of the anomaly cancellation in M-theory on orbifolds $S^{1} / \mathbb{Z}_{2}$ and $T^{5} / \mathbb{Z}_{2}$ in the upstairs approach. The main requirement that allows one to uniquely define solutions to the modified Bianchi identities in this case is that the field strength $G$ be globally defined on $S^{1}$ or $T^{5}$ and properly transforming under $\mathbb{Z}_{2}$. We solve for general $G$ that satisfies these requirements and explicitly construct anomaly-free theories in the upstairs approach. We also obtain the solutions in the presence of five-branes. All these constructions show equivalence of the downstairs and upstairs approaches. For example in the $S^{1} / \mathbb{Z}_{2}$ case the ten-dimensional gauge coupling and the anomaly cancellation at each wall are the same as in the downstairs approach.
\end{abstract}

March 2000 


\section{Introduction}

Dualities in string theory connect seemingly different theories. One of the most unexpected predictions in this web of dualities is the appearance of 11-dimensional theory (M-theory) that manifests itself at low energies as 11-dimensional supergravity. The theory compactified on different spaces is dual to different string theories. Compactification on $S^{1} / \mathbb{Z}_{2}$ gives the M-theoretic extension of the heterotic $E_{8} \times E_{8}$ string, i.e. a geometric picture of 11-dimensional spacetime with two 10-dimensional walls at the ends of a finite interval along eleventh dimension [1, 2, 3]. While the supergravity multiplet (graviton, gravitino and antisymmetric tensor fields) can penetrate in the $d=11$ bulk, the two $E_{8}$ gauge supermultiplets are confined to the two walls, respectively. The requirement of anomaly cancellation gives the relation between the 11-dimensional Newton's constant $\kappa$ and the 10-dimensional gauge coupling constant $\lambda$ - it was first obtained in [2] with a numerical factor of 2 missing. The correct relation was first obtained in [4] and subsequently by many authors both in the downstairs and in the upstairs approaches [5, 6]. However, as was noted in [7], the previous calculations in the upstairs approach were inconsistent because of incorrect taking into account the $\mathbb{Z}_{2}$ symmetry. Rather surprisingly, the result of the upstairs calculation of [7] gave different result than the downstairs calculation of [4].

Another interesting example of an orbifold in M-theory compactification is $T^{5} / \mathbb{Z}_{2}$ [8, 9] which is dual to string theory IIB compactified on $K 3$. There are 32 fixed sixplanes and it turns out that there must be 16 additional twisted sector multiplets living on these six-planes to cancel anomalies. In the presence of five-branes some of these tensor multiplets are transferred to the branes.

In section 2 we analyze the anomaly cancellation in the upstairs approach for the compactification on $S^{1} / \mathbb{Z}_{2}$ and the results show complete equivalence of the downstairs and the upstairs approaches. The gauge anomaly is restricted to the walls and there are no mixing terms between the walls so that the cancellation proceeds exactly as in the downstairs approach. The combination $\lambda^{6} / \kappa^{4}$ turns out to be exactly the same as in [4] (while in ref. [7] it differs by a factor of 3). In section 3 we include five-branes 
and derive the field configuration for which the total anomaly vanishes. In section 4 we explicitly construct a theory compactified on $T^{5} / \mathbb{Z}_{2}$ and show that there exists in the upstairs approach a configuration of fields that is locally (at each fixed six-plane) anomaly-free. Section 5 presents some conclusions.

\section{M-theory on $S^{1} / \mathbb{Z}_{2}$}

The low energy limit of M-theory compactified on $S^{1} / \mathbb{Z}_{2}$ was given in [1, 2]. We will use in this paper slightly different normalization of the fields (the same as in [7]). The actions for the theory in the upstairs and downstairs approaches look the same but the range of integration and the normalizations are different.

In the upstairs approach we have full circle (of length $2 \pi \rho$ ) in the eleventh dimension with two branes located at $x^{11}=0$ and $x^{11}=\pi \rho$ and we impose $\mathbb{Z}_{2}$ symmetry on fields (for example $C_{A B C}$ and $G_{A B C D}$ with $A, B, C, D=1 \ldots 10$ are odd under $\mathbb{Z}_{2}$ and therefore vanish on the walls while $C_{11 A B}$ and $G_{11 A B C}$ are even). The action reads (for simplicity we keep only bosonic terms)

$$
S=-\frac{1}{2 \bar{\kappa}^{2}} \int_{\bar{M}_{11}} \sqrt{-g}\left[R+\frac{1}{48} G^{2}\right]-\frac{1}{12 \bar{\kappa}^{2}} \int_{\bar{M}_{11}} C \wedge G \wedge G-\frac{1}{4 \lambda^{2}} \sum_{i} \int_{M_{10}^{i}} \sqrt{-g} F_{i}^{2}
$$

In the downstairs approach we take an interval (of length $\pi \rho$ ) with the two walls on

its ends and instead of the $\mathbb{Z}_{2}$ symmetry we have to impose appropriate boundary conditions. The action is the same but we have to replace $\bar{M}_{11}$ by $M_{11}$ (full circle by the interval in the eleventh dimension) and the coupling constant $\bar{\kappa}$ by $\kappa$ where:

$$
\kappa^{2}=\frac{1}{2} \bar{\kappa}^{2}, \quad \int_{M_{11}}=\frac{1}{2} \int_{\bar{M}_{11}} .
$$

Anomaly cancellation requires modifications of the Bianchi identities that involve sources on the walls. We will solve below for general $G$ and $C$ that satisfy these identities and are well defined on the full circle (i.e. are periodic).

Let us start with some definitions that will prove useful later on. We introduce a space $\Phi$ of differentiable functions defined on the interval $[0, \pi \rho]$ and satisfying

$$
g \in \Phi: \quad g(0)=1, \quad g(\pi \rho)=0 .
$$


For any $g \in \Phi$ we define two periodic functions, $f_{g}^{(1)}$ and $f_{g}^{(2)}$, defined on the circle $(-\pi \rho, \pi \rho]:$

$$
\begin{aligned}
& f_{g}^{(1)}\left(x^{11}\right)=\operatorname{sgn}\left(x^{11}\right) g\left(\left|x^{11}\right|\right), \\
& f_{g}^{(2)}\left(x^{11}\right)=\operatorname{sgn}\left(x^{11}\right)\left(g\left(\left|x^{11}\right|\right)-1\right), \\
& f_{g}^{(1)}(\pi \rho)=f_{g}^{(2)}(\pi \rho)=0 .
\end{aligned}
$$

These functions constitute the most general $\mathbb{Z}_{2}$-odd primitives of delta functions located, respectively, at 0 and $\pi \rho$, having no other singularities and defined globally on the circle. The derivatives of these functions are:

$$
\frac{\partial f_{g}^{(i)}}{\partial x^{11}}=2 \delta^{(i)}+h_{g}
$$

where $h_{g}\left(x^{11}\right)=g^{\prime}\left(\left|x^{11}\right|\right)$ is regular everywhere and $\delta^{(i)}$ is the Dirac delta function located at the $i$-th wall. Later we will use the same symbol to denote the corresponding one form. It is easy to prove that for any $g$

$$
\int_{S_{1}} d x^{11} h_{g}\left(x^{11}\right) f_{g}^{(i)}\left(x^{11}\right) f_{g}^{(j)}\left(x^{11}\right)=\frac{1}{3}-\delta_{i j}
$$

Regularization of the delta function gives also:

$$
\begin{aligned}
\delta^{(i)} f_{g}^{(j)} f_{g}^{(k)} & \rightarrow \frac{1}{3}\left(\delta_{i j} \delta_{i k}\right) \delta^{(i)} \\
\delta^{(i)} f_{g}^{(j)} & \rightarrow 0
\end{aligned}
$$

(in sense of distributions i.e. when integrated with regular functions).

Having defined the primitives of delta functions we can now solve for $G$ in the modified Bianchi identities. They read

$$
d G=-\gamma \sum_{i} \delta^{(i)} \wedge I_{i}
$$

where $\gamma=(4 \pi)^{2} \bar{\kappa}^{2} / \lambda^{2}$ and

$$
I_{i}=\left.\frac{1}{4 \pi^{2}}\left(\operatorname{tr} F_{i}^{2}-\frac{1}{2} \operatorname{tr} R^{2}\right)\right|_{M_{10}^{i}} .
$$

An integral of $d G$ over a $C_{4} \times I$ where the interval $I$ surrounds only one wall is not vanishing but the integral over the entire interval should vanish for $G$ to be well defined. Therefore the sum of $I_{1}$ and $I_{2}$ must be cohomologically trivial:

$$
\int_{C_{4}}\left(I_{1}+I_{2}\right)=0 \text {. }
$$


Because of (11) there exists a form of $G$ that is explicitly well defined globally. We can write

$$
\begin{aligned}
& I_{1}=H_{4}+d \Omega_{1}, \\
& I_{2}=-H_{4}+d \Omega_{2}
\end{aligned}
$$

where $H_{4}$ is a harmonic 4-form and $\Omega_{i}$ are 3-forms well defined globally. Then we can write the solution of (9) in the form

$$
G=d \tilde{C}-\frac{\gamma}{2} \operatorname{sgn}\left(x^{11}\right) H_{4}+\gamma \sum_{i} \delta^{(i)} \wedge \Omega_{i}
$$

where each of the terms is well defined globally. Unfortunately, we do not know the explicit form of (12) and it is difficult to connect $\tilde{C}$ with the $C$ field from M-theory where $G=d C$ in the bulk. Therefore we will seek the solution of (9) in a different form which is only implicitly well defined globally. The solution to (9) satisfying $G=d C$ in the bulk is given by

$$
G=d C+\gamma \sum_{i} \delta^{(i)} \wedge \omega_{i}
$$

where $I_{i}=d \omega_{i}$ locally円. In order to analyze the consequences of $\mathbb{Z}_{2}$ symmetry, we will take care to define all forms globally at least in the eleventh dimension. We expect $G$ to be a regular form and therefore we expect that singularities in $\delta C$ should cancel $\delta^{(i)}$ in (14). Let us define a regular three-form $C_{r e g}$ (depending on functions $g_{1}$ and $g_{2}$ ) by the relation

$$
C=C_{r e g}-\frac{\gamma}{2} \sum_{i} f_{g_{i}}^{(i)} \omega_{i}
$$

Substituting this form to (14) we get a regular (along $x^{11}$ ) form

$$
G=d C_{r e g}-\frac{\gamma}{2} \sum_{i} h_{g_{i}} d x^{11} \wedge \omega_{i}-\frac{\gamma}{2} \sum_{i} f_{g_{i}}^{(i)} I_{i}
$$

where we used (5).

Integration of Bianchi identities over $I \times C_{4}$ where $I$ is an interval along $x^{11}$ comprising only one wall shows that $G$ in (16) is globally well defined only if

$$
g_{1}\left(x^{11}\right)=g_{2}\left(x^{11}\right)=g\left(x^{11}\right) .
$$

\footnotetext{
${ }^{1}$ In general, $\omega_{i}$ is not well defined globally since $I_{i}$ can have contributions from harmonic forms $\left(H_{4}\right.$ in (12) ) but $C$ is likewise not well defined globally and the two contributions should cancel to produce well defined $G$ like in (13).
} 
Hence, the same function has to be used in $f_{g}^{(1)}$ and $f_{g}^{(2)}$ (and consequently there is only one regular derivative $h$ ). Therefore, we will suppress subscript $g$ from now on.

To calculate all contributions to the anomaly, we have first to find gauge variation of $C$. Starting from the condition $\delta G=0$ and using equations (14) and (15) we get

$$
\delta C_{r e g}=d B_{r e g}-\frac{\gamma}{2} h \sum_{i} d x^{11} \wedge \omega_{i}^{1}
$$

where $\delta \omega_{i}=d \omega_{i}^{1}$.

Let us now calculate the anomaly in the upstairs approach. The contribution from the topological term in the action is equal to

$$
\delta S_{\text {top }}=-\frac{1}{12 \bar{\kappa}^{2}} \int \delta C \wedge G \wedge G .
$$

The relation $G=d C$ valid in the bulk requires $C$ (and not $C_{r e g}$ ) in the above expression - we would break supersymmetry in the bulk otherwise. Let us note that the authors of [7] used $\delta C_{r e g}$ in (19) and not $\delta C$ and it is one of the reasons for the difference between the results of the present paper and that of $[7]^{2}$.

Using the expressions (16) and (18) we have

$$
\begin{aligned}
\delta C & =d B_{r e g}-\frac{\gamma}{2} h \sum_{i} d x^{11} \wedge \omega_{i}^{1}-\frac{\gamma}{2} \sum_{i} f^{(i)} d \omega_{i}^{1} \\
G & =d C_{r e g}-\frac{\gamma}{2} h \sum_{i} d x^{11} \wedge \omega_{i}-\frac{\gamma}{2} \sum_{i} f^{(i)} I_{i} .
\end{aligned}
$$

It is easy to show (see relation (8)) that the terms with $d B_{\text {reg }}$ and $d C_{\text {reg }}$ do not contribute to (19) due to regularity of $B_{r e g}$ and $C_{r e g}$ and the fact that $\left.G_{A B C D}\right|_{i}=0$. Therefore we get

$$
\begin{aligned}
\delta S_{\text {top }}= & \frac{1}{12 \bar{\kappa}^{2}}\left(\frac{\gamma}{2}\right)^{3} \int_{\bar{M}_{11}} \sum_{i j k}\left(h d x^{11} \wedge \omega_{i}^{1}+f^{(i)} d \omega_{i}^{1}\right) \wedge \\
= & \frac{\gamma^{3}}{96 \bar{\kappa}^{2}} \sum_{i j k}\left(\int_{S_{1}} h f^{(j)} f^{(k)} \int_{M_{10}} \omega_{i}^{1} \wedge \omega_{j} \wedge f^{(j)} I_{j}\right) \wedge\left(h d I_{k}^{11} \wedge \omega_{k}+f^{(k)} I_{k}\right) \\
& \left.\quad-2 \int_{S_{1}} h f^{(i)} f^{(k)} \int_{M_{10}} d \omega_{i}^{1} \wedge \omega_{j} \wedge I_{k}\right) \\
= & \frac{\gamma^{3}}{96 \bar{\kappa}^{2}} \sum_{i j k} \int_{M_{10}}\left[\left(\frac{1}{3}-\delta_{j k}\right) \omega_{i}^{1} \wedge I_{j} \wedge I_{k}+2\left(\frac{1}{3}-\delta_{i k}\right) \omega_{i}^{1} \wedge I_{j} \wedge I_{k}\right]
\end{aligned}
$$

\footnotetext{
${ }^{2}$ In [7] a linear function was chosen for $g$ but as we show in the present paper nothing really depends on the choice of $g$ as long as conditions (3) are satisfied.
} 
Expanding this result shows that the terms which mix contributions from different walls cancel and we have the final result

$$
\delta S_{\text {top }}=-\frac{\gamma^{3}}{48 \bar{\kappa}^{2}} \sum_{i} \int_{M_{10}} \omega_{i}^{1} \wedge I_{i} \wedge I_{i} .
$$

The next contribution to the anomaly is the Green-Schwarz term - we take it to be of the form? 2, 10,

$$
S_{G S}=-\frac{1}{\gamma} \int_{\bar{M}_{11}} C \wedge X_{8}
$$

Calculating the gauge variation of this term using (20) we get

$$
\begin{aligned}
\delta S_{G S} & =\frac{1}{2} \int_{\bar{M}_{11}} \sum_{i}\left(h d x^{11} \wedge \omega_{i}^{1}+f^{(i)} d \omega_{i}^{1}\right) \wedge X_{8} \\
& =-\int_{\bar{M}_{11}} \sum_{i} \delta^{(i)} \wedge \omega_{i}^{1} \wedge X_{8}=-\sum_{i} \int_{M_{10}} \omega_{i}^{1} \wedge X_{8, i} .
\end{aligned}
$$

The third contribution to the anomaly is the one-loop result - it is given by

$$
\delta S_{1-\text { loop }}=\frac{\pi}{3} \sum_{i} \int_{M_{10}} \omega_{i}^{1} \wedge I_{i} \wedge I_{i}+\sum_{i} \int_{M_{10}} \omega_{i}^{1} \wedge X_{8, i}
$$

The requirement of vanishing of the total anomaly i.e the sum of (23), (25) and (26) gives

$$
\frac{\lambda^{6}}{\bar{\kappa}^{4}}=\frac{(4 \pi)^{5}}{4}
$$

Translating this result to the downstairs language using (2) we get

$$
\frac{\lambda^{6}}{\kappa^{4}}=(4 \pi)^{5}
$$

and this relation is exactly the same as in 4 .

\section{M-theory on $S^{1} / \mathbb{Z}_{2}$ with five-branes}

Let us now include five-branes - possible non-perturbative objects in M-theory that couple magnetically to $G$ and act as sources for modified Bianchi identities. Their

\footnotetext{
${ }^{3}$ Choosing instead $G \wedge X_{7}$ with $X_{8}=d X_{7}$ would give the same contribution to the anomaly expressed as 12-form but the anomaly cancellation in the 10-form would then require a local counterterm.
} 
presence can in general modify the condition (11) and therefore require non-standard identification of gauge fields with the connection. We would like to show here that we can repeat the whole discussion in the presence of five-branes in the upstairs approach.

Let us take into account the five-branes parallel to three space dimensions and two CY dimensions (it is possible that with the non-standard embedding it is no longer a Calabi-Yau space but let us leave this subtlety aside). These five-branes act then as additional sources in the Bianchi identity

$$
d G=-\gamma \sum_{i} \delta^{(i)} \wedge I_{i}-\gamma \sum_{\alpha} \delta_{5}^{(\alpha)}
$$

where $\delta_{5}^{(\alpha)}$ are products of five delta one forms along four CY dimensions and $x^{11}$. The requirement that $G$ be globally defined gives

$$
\int_{C_{4}}\left(I_{1}+I_{2}\right)+\left[C_{\alpha}\right]=0
$$

where $\left[C_{\alpha}\right]$ is equal to the number of five-branes surrounded by a given cycle $C_{4}$.

In analogy to the previous case let us introduce a $\mathbb{Z}_{2}$ odd and periodic function with jumps at $x^{11}=x_{\alpha^{+}}>0$ and $x^{11}=-x_{\alpha^{+}}$(it necessarily has two jumps symetrically located around $x^{11}=0$ because of $\mathbb{Z}_{2}$ antisymmetry):

$$
f^{(\alpha)}\left(x^{11}\right)=\operatorname{sgn}\left(x^{11}\right)\left[g\left(\left|x^{11}\right|\right)-\theta\left(x_{\alpha^{+}}^{11}-\left|x^{11}\right|\right)\right]
$$

where $\theta$ is the Heaviside step function.

Solving for $G$ gives in analogy to (16)

$$
G=d C_{r e g}-\frac{\gamma}{2} \sum_{i} h d x^{11} \wedge \omega_{i}-\frac{\gamma}{2} \sum_{i} f^{(i)} I_{i}-\gamma \sum_{\alpha^{+}} h d x^{11} \wedge \theta_{3}^{(\alpha)}-\gamma \sum_{\alpha^{+}} f^{(\alpha)} d x^{11} \wedge \delta_{4}^{(\alpha)}
$$

where $d \theta_{3}^{(\alpha)}=\delta_{4}^{(\alpha)}$. Therefore, we have to sum in (32) only over positive $x_{\alpha}$ (denoted by $\alpha^{+}$) since the branes at negative values of $x^{11}$ are automatically taken into account. A similar argument as before (integration over a cycle comprising only one brane) shows that the function $g$ in (31) is the same for all branes and also the same as the one defining $f^{(1)}$ and $f^{(2)}$.

The condition (30) that $G$ be globally defined is also crucial for anomaly cancellation and for confining anomaly to the wall or brane. Let us now describe the anomaly 
cancellation in the case with five-branes. We will work with 8-forms (and not 6-forms) and will not keep track of possible local counterterms.

The contribution to the anomaly from the one-loop result is

$$
\delta S^{1-l o o p}=\sum_{\alpha} X_{8, \alpha}
$$

The contribution to the anomaly from the Green-Schwarz mechanism is

$$
-\frac{1}{\gamma} \delta \int G \wedge X_{7}=-\frac{1}{\gamma} \int G \wedge d X_{6}^{1}=-\sum_{\alpha} X_{6, \alpha}^{1}
$$

The contribution (34) translated to the 8-form language cancels the contribution from (33). Therefore, we expect that the contribution from the topological term should vanish. Let us show that (under some condition) this indeed is the case. Since $\delta_{5}$ is invariant under gauge and gravitational transformations, $\delta C$ is the same as before (20):

$$
\begin{aligned}
\delta C & =d B_{\text {reg }}-\frac{\gamma}{2} h \sum_{i} d x^{11} \wedge \omega_{i}^{1}-\frac{\gamma}{2} \sum_{i} f^{(i)} d \omega_{i}^{1} \\
& =d\left(B_{\text {reg }}-\frac{\gamma}{2} \sum_{i} f^{(i)} \omega_{i}^{1}\right)+\gamma \sum_{i} \delta^{(i)} \wedge \omega_{i}^{1} .
\end{aligned}
$$

Using (29) we get the five-brane contribution to the anomaly

$$
\left.\int \delta C \wedge G \wedge G\right|_{f b}=2 \gamma \int \sum_{\alpha}\left(B_{r e g}-\frac{\gamma}{2} \sum_{i} f^{(i)} \omega_{i}^{1}\right) \wedge\left(d C_{r e g}-\frac{\gamma}{2} \sum_{j} f^{(j)} I_{j}\right) \wedge \delta_{4}^{(\alpha)}
$$

Therefore, if we impose conditions for all positions of branes $\left(x_{\alpha}^{11}\right)$ :

$$
B_{r e g}\left(x_{\alpha}^{11}\right)=\frac{\gamma}{2} \sum_{i} f^{(i)}\left(x_{\alpha}^{11}\right) \omega_{i}^{1}
$$

then the contribution to the anomaly from the topological term vanishes and summing up all contributions the total anomaly does so as well.

\section{M-theory on $T^{5} / \mathbb{Z}_{2}$}

Another interesting orbifold compactification of $\mathrm{M}$-theory is that on $T^{5} / \mathbb{Z}_{2}$ i.e. down to a theory in six dimensions with 16 supercharges [8, 9, 6]. Similarly as in the ten dimensional case there is a potential gravitational anomaly. In the limit of small 
compactification radius the particle content of this theory (after imposing $\mathbb{Z}_{2}$ on the fields) is a chiral supergravity multiplet (consisting of a graviton, 2 chiral gravitinos and 5 selfdual twoforms) and five tensor multiplets (each consisting of an antiselfdual twoform, 2 antichiral fermions and 5 scalars). This theory does not possess vectors so that the potential anomaly must be purely gravitational. It turns out that the anomaly vanishes when there are additional 16 tensor multiplets (so called twisted sector not directly obtained from the compactification). $T^{5} / \mathbb{Z}_{2}$ has 32 fixed six-planes and the additional matter multiplets should live on these six-planes. The anomaly from the small radius theory (untwisted sector only) is equally distributed among all 32 points so that the anomaly at the $P$-th plane is given by:

$$
I_{8, P}^{(1-l o o p)}=\frac{1}{32}\left(2 I^{(3 / 2)}-10 I^{(1 / 2)}\right)+N_{P}\left(-2 I^{(1 / 2)}-I^{(3-\text { form })}\right)
$$

where $N_{P}$ is the number of the twisted sector matter multiplets living on the $P$-th fixed plane. Using explicit formulae for the anomalies in six dimensions one gets

$$
I_{8, P}^{(1-l o o p)}=\left(N_{P}-\frac{1}{2}\right) X_{8}
$$

Sum over $P$ gives the previously mentioned condition $\sum N_{P}=16$. Since $N_{P}$ are integers it is impossible to cancel the anomaly at any fixed six-plane and to do so one has to modify Bianchi identities to provide additional source of anomaly inflow via Green-Schwarz mechanism. If

$$
d G=\sum_{P} g_{P} \delta_{5}^{(P)}
$$

then by the same arguments as before the full anomaly is given by

$$
I_{8, P}=\left(N_{P}-g_{P}-\frac{1}{2}\right) X_{8}
$$

To cancel the anomaly the magnetic charges have to be half-integers satisfying

$$
g_{P}=N_{P}-\frac{1}{2}
$$

We will describe below an explicit construction of $G$ satisfying (40) in the upstairs approach. We could apply the techniques worked out in the case of $S^{1} / \mathbb{Z}_{2}$ but it is not necessary. The $T^{5} / \mathbb{Z}_{2}$ orbifold is simpler because the cohomology condition (30) is now replaced by an algebraic relation $\sum N_{P}=16$. 
Let us denote $2^{5}=32$ fixed six-planes by $P(\bar{n})$ where $\bar{n}=\left(n_{7}, n_{8}, n_{9}, n_{10}, n_{11}\right)$ and $n_{k}=0,1$. The action of $\mathbb{Z}_{2}$ on the torus $\otimes_{k}\left(-\pi \rho_{k}, \pi \rho_{k}\right]$ that leaves these 32 points intact is given by $x_{i} \rightarrow-x_{i}$. Let us also introduce $5 \times 2^{4}=80$ intervals joining fixed points by $I\left(\bar{n}^{k}\right)$ where $\bar{n}^{k}=\left(n_{7}, \ldots, n_{11}\right)$ with $n_{k}$ left out. The interval $I\left(\bar{n}^{k}\right)$ is parallel to the $k$-th axis and we assume that its orientation is the same as the orientation of that axis.

The field $G$ satisfying (40) and antisymmetric under $\mathbb{Z}_{2}$ is given by

$$
G=d C+\frac{1}{2} \sum_{k} \sum_{\bar{n}^{k}} c\left(\bar{n}^{k}\right) \operatorname{sgn}\left(x^{k}\right) \delta_{4}^{\left(\bar{n}^{k}\right)}
$$

where

$$
\delta_{4}^{\left(\bar{n}^{k}\right)}=\frac{1}{4 !} \epsilon_{k p q r s} \delta\left(x^{p}-n_{p} \pi \rho_{p}\right) \wedge \ldots \wedge \delta\left(x^{s}-n_{s} \pi \rho_{s}\right)
$$

and $c\left(\bar{n}^{k}\right)$ are constants characterizing $G$ along the interval $I\left(\bar{n}^{k}\right)$. Calculating $d G$ from (43) and comparing with (40) and (42) we get

$$
g_{P(\bar{n})}=\sum_{k}(-1)^{n_{k}} c\left(\bar{n}^{k}\right)=N_{P}-\frac{1}{2} .
$$

In the above equation $\bar{n}^{k}$ has the same components as $\bar{n}$ but with $n_{k}$ dropped. It is straightforward for any given set of $N_{P}$ (satisfying $\sum N_{P}=16$ i.e. $\sum g_{P}=0$ ) to get $c\left(\bar{n}^{k}\right)$ satisfying the above equation. Let us describe explicitly for example the "checkerboard" configuration [8] where 16 tensor multiplets are distributed in the most uniform way:

$$
N_{P}=\frac{1}{2}\left[1+(-1)^{n_{7}+n_{8}+n_{9}+n_{10}+n_{11}}\right]
$$

Shrinking any of the radii in the above configuration, any pair of fixed points along the shrinking direction contributes 1 tensor multiplet what corresponds to the string limit [8, 9]. For this choice for $N_{P}$ it is easy to derive one of possible sets $c\left(\bar{n}^{k}\right)$ :

$$
c\left(\bar{n}^{k}\right)=\frac{1}{10}(-1)^{n_{7}+\ldots n_{11}}
$$

(where the index $n_{k}$ is left out on the r.h.s.) and check that (45) is satisfied. There are of course other $c\left(\bar{n}^{k}\right)$ - differring by $d C$ in (43) - that give the same local cancellation of anomalies. 
Other distributions of tensor multiplets among the fixed planes can be obtained from the "checkerboard" configuration by moving those mutliplets one by one from some fixed point to the neighbouring one along interval $I\left(\bar{n}^{k}\right)$. Such change requires appropriate modification of $c\left(\bar{n}^{k}\right)$ :

$$
\Delta c\left(\bar{n}^{k}\right)= \pm \frac{1}{2}
$$

where the sign depends on the "orientation" of the exchange.

The theory in the presence of five-branes orthogonal to $T^{5}$ can be similarly analyzed. The location of the $\alpha$-th five-brane is given by a vector with components $x_{\alpha}^{k}$ for $k=$ $7, \ldots, 11$. In the presence of such five-branes the modified Bianchi identities read

$$
d G=\sum_{P} g_{P} \delta_{5}^{(P)}+\sum_{\alpha} \delta_{5}^{(\alpha)}
$$

Because of the $\mathbb{Z}_{2}$ symmetry the five-branes must come in pairs with opposite coordinates: $x_{\beta}^{k}=-x_{\alpha}^{k}$. In order to satisfy (49) the field $G$ must have jumps at the location of both five-branes. The requirement that $G$ is globally well defined can be fulfilled only when there are two additional jumps of opposite sign located at some fixed six-planes. Such negative jumps of $G$ at the fixed planes correspond to removing two twisted sector matter multiplets from those fixed planes and transfering them to the so called "wandering" five-branes. One of the solutions is to put both of those negative jumps for all pairs of five-branes at the origin $P(\bar{n}=(0,0,0,0,0))$. If we want to transfer tensor multiplets from some other fixed six-planes we have first to move those multiplets to the origin using the procedure described before (48). The solution transferring all multiplets from the origin to the branes is given by the sum of (43) and

$$
\Delta G=-\frac{1}{2 \cdot 5 !} \sum_{\alpha} \sum_{p q r s t} \epsilon_{p q r s t} \operatorname{sgn}\left(x^{p}\right) \theta\left(\left|x_{\alpha}^{p}\right|-\left|x^{p}\right|\right) \delta\left(x^{q}-x_{\alpha}^{q}\right) \wedge \ldots \wedge \delta\left(x^{t}-x_{\alpha}^{t}\right) .
$$

Now 16 twisted sector tensor multiplets are distributed between fixed planes and fivebranes

$$
\sum_{P} N_{P}+\sum_{\alpha} 1=16
$$

The extremal situation is reached when all tensor multiplets sit on five-branes and there are no twisted sector multiplets on the fixed six-planes. 


\section{Conlusions}

We have analyzed the problem of anomaly cancellation in the upstairs approach. It turned out that for the compactification on $S^{1} / \mathbb{Z}_{2}$ orbifold all final results are sums of contributions from two walls so the anomaly cancellation can be achieved without any corrections to the original action. $C$ and $G$ fields depend on an arbitrary function of $x^{11}$ but the anomaly cancellation works in the same way for any choice of this function (however, one should note that for other purposes some choice may be preferred over the others like for example in [11] where it was shown that the Kaluza-Klein zero modes have some specific dependence on the eleventh dimension). The cancellation of anomalies in the presence of five-branes is possible but requires one additional condition on the variation of $C$ under the gauge and gravitational transformations. In the case of compactification on $T^{5} / \mathbb{Z}_{2}$ (with and without five-branes) we have presented in the upstairs approach an explicit method to derive field configuration for which the total anomaly vanishes separately at each fixed six-plane.

The same results can be obtained in the downstairs approach but with appropriate boundary conditions replacing the $\mathbb{Z}_{2}$ symmetry. This, however, is less straightforward to implement. For example the upstairs approach automatically takes into account proper normalization of charges. In the case of the $T^{5} / \mathbb{Z}_{2}$ compactification the number of additional tensor multiplets is always 16 in the upstairs approach (since two $\mathbb{Z}_{2}$ symmetric "wandering branes" carry two tensor multiplets) while in the downstairs approach it can be smaller (because now there is only a single brane with one tensor multiplet). In conclusion, from the point of view of anomaly cancellation, the upstairs and downstairs approaches are equivalent. In the actual computation, however, the upstairs approach seems to be more convenient.

\section{Acknowledgements}

We would like to thank J. Conrad and H.P. Nilles for discussions. K.A.M. was partially supported by the Polish KBN grant 2P03B 03715 (1998-2000). M.O. was partially supported by the Polish grant KBN 2 P03B 05216 (1999-2000). 


\section{References}

[1] P. Hořava and E. Witten, Heterotic and type I string dynamics from eleven dimensions, Nucl. Phys. B460 (1996) 506.

[2] P. Hořava and E. Witten, Eleven-Dimensional Supergravity on a Manifold with Boundary, Nucl. Phys. B475 (1996) 94.

[3] E. Witten, Strong Coupling Expansion Of Calabi-Yau Compactification, Nucl. Phys. B471 (1996) 135.

[4] J.O. Conrad, Brane tensions and coupling constants from within M-theory, Phys. Lett. B421 (1998) 119.

[5] J.X. Lu, Remarks on M-theory coupling constants and M-brane tension quantizations, hep-th/9711014; T. Harmark, Coupling constants and brane tensions from anomaly cancellation in M-theory, Phys. Lett. B431 (1998) 295; M. Faux, Confluences of anomaly freedom requirements in M-theory, hep-th/9803254;

[6] M. Faux, D. Lüst and B.A. Ovrut, Intersecting orbifold planes and local anomaly cancellation in M-theory, Nucl. Phys. B554 (1999) 437.

[7] A. Bilal, J.-P. Derendinger, R. Sauser, M-theory on $S^{1} / Z_{2}$ : Facts and Fakes, hep-th/9912150.

[8] E. Witten, Five-branes and M-theory on an orbifold, Nucl. Phys. B463 (1996) 383.

[9] K. Dasgupta and S. Mukhi, Orbifolds of M-theory, Nucl. Phys. B465 (1996) 399.

[10] C. Vafa and E. Witten, A one loop test of string duality, Nucl. Phys. B447 (1995) 261; M.J. Duff, J.T. Liu and R. Minasian, Eleven-dimensional origin of string/string duality: A one-loop test, Nucl. Phys. B452 (1995) 261.

[11] K.A. Meissner, H.P. Nilles and M. Olechowski, Supersymmetry breakdown at distant branes: the super-Higgs mechanism, Nucl. Phys. B561 (1999) 30. 